\title{
Problems and limitations in thrombolysis of acute stroke patients at a tertiary care center
}

\author{
Sushma K. Gurav, Kapil G. Zirpe ${ }^{3}$, R. S. Wadia', Manishprasad K. Pathak, Abhijeet M. Deshmukh, \\ Rahul V. Sonawane ${ }^{2}$, Nikhil Goli
}

Aim: (I) To evaluate the number of patients thrombolysed within I $\mathrm{h}$ of arrival to emergency room (ER) (2) To identify reasons for delay in thrombolysis of acute stroke patients. Materials and Methods: All patients admitted to ER with symptoms suggestive of stroke from January $201 \mathrm{I}$ to November 2013 were studied. Retrospective data were collected to evaluate ER to needle (door to needle time [DTNt]) time and reasons for delay in thrombolysis. The parameters studied (I) onset of symptoms to ER time, (2) ER to imaging time (door to imaging time [DTIt]), (4) ER to needle time (door to needle) and (5) contraindications for thrombolysis. Results: A total of 695 patients with suspected stroke were admitted during study period. 547 (78\%) patients were out of window period. I 48 patients $(21 \%, M=104, F=44)$ arrived within window period $(<4.5$ h.). 104 (70.27\%) were contraindicated for thrombolysis. Majority were intracerebral bleeds. $44(29.7 \%)$ were eligible for thrombolysis.7 (I5.9\%) were thrombolysed within I h.The mean time for arrival of patients from onset of symptoms to hospital (symptom to door) 83 min (median - 47). The mean door to neuro-physician time (DTPt) was $32 \mathrm{~min}$ (median - $15 \mathrm{~min}$ ). The mean DTIt was 58 min (median - 50 min). The mean DTNt 104 (median - 100 min). Conclusion: Reasons for delay in thrombolysis are:Absence of stroke education program for common people. Lack of priority for triage and imaging for stroke patients.

Keywords: Acute ischemic stroke, window period, thrombolysis triage and stroke code team

\section{Introduction}

Thrombolysis with recombinant tissue plasminogen activator (rtPA, alteplase) is the only effective specific treatment for acute ischemic stroke patients coming in window (4.5 h from onset of symptoms). ${ }^{[1]}$ A milestone study of National Institute of Neurological Disorders and Stroke in 1995, demonstrated the benefits of rtPA in to patients of acute ischemic stroke (AIS) who came within window period. These patients of AIS who were thrombolysed were $30 \%$ more likely to survive with

From:

Intensivist, Neuro-Trauma Unit, Ruby Hall Clinic, ${ }^{3}$ Director, Neuro-Trauma Unit, Ruby Hall Clinic, ${ }^{1}$ Director, Neuro-Sciences, Ruby Hall Clinic, ${ }^{2}$ Chief Resident, Medicine, Ruby Hall CInic, Pune, India

Correspondence:

Dr. Sushma K. Gurav,

Neuro-Trauma Unit, Ruby clinic,

Pune, Maharashtra - 411001 , India.

E-mail: kirtisush_gurav@yahoo.co.in minimal disability resulting in a $12 \%$ absolute increase in the proportion having excellent functional outcomes at 3 months. ${ }^{[2]}$ After publication of the European Cooperative Acute. Stroke Study III trail (2008) window period was extended to $4.5 \mathrm{~h}$. For every $15 \mathrm{~min}$ reduction in door to needle time (DTNt) there is $5 \%$ reduction in odds of in hospital mortality (odd ratio, $0.95 ; 95 \%$ confidence interval, 0.92-0.98: $P=0.0007) \cdot{ }^{[3]}$

\section{Materials and Methods}

This is retrospective study which was approved by Institutional ethical board committee. All patients who presented with stroke to emergency department (ER) from January 2011 to December 2013 were included in the study. After initial assessment by causality personnel a medical/neuro-resident evaluated the patient. Radiological diagnosis was obtained with noncontrasted brain computed tomography and/or diffusion weighted 
magnetic resonance imaging (DWI). Images were evaluated by a radiologist. After neuro physician opinion or after telephonic discussion by neuro-resident with neuro physician (telestroke) the treatment plan was decided. For the patients who presented within window period thrombolysis was planned. Severity of Stroke was documented by The National Institutes of Health Stroke Scale (NIHSS). Risk factor for stroke were recorded. Contra-indications for thrombolysis were checked and consent of relatives/patient was taken. Those patients who presented out of window period $(>4.5 \mathrm{~h}$ after onset of symptom/symptom to door time [STD] $>4.5 \mathrm{~h}$ ), or patients who had hemorrhagic stroke and those who were not willing for giving consent were excluded [Figure 1].

Patients record files and charts were used to extract retrospective data. The collected data use to evaluate ER to needle [door to needle time-(DTNt)] time and reasons for delay in thrombolysis therapy in acute stroke patients.

The following parameters were studied

- Onset of symptoms to ER time,

- Assessment by physician/medical chief resident time (door to physician time [DTPt])

- ER to imaging time (door to imaging time [DTIt]),

- ER to needle time (DTNt)

- Contraindications for thrombolysis.

The onset of symptom time for patients with wake up was accepted as last time the patient was seen as healthy.

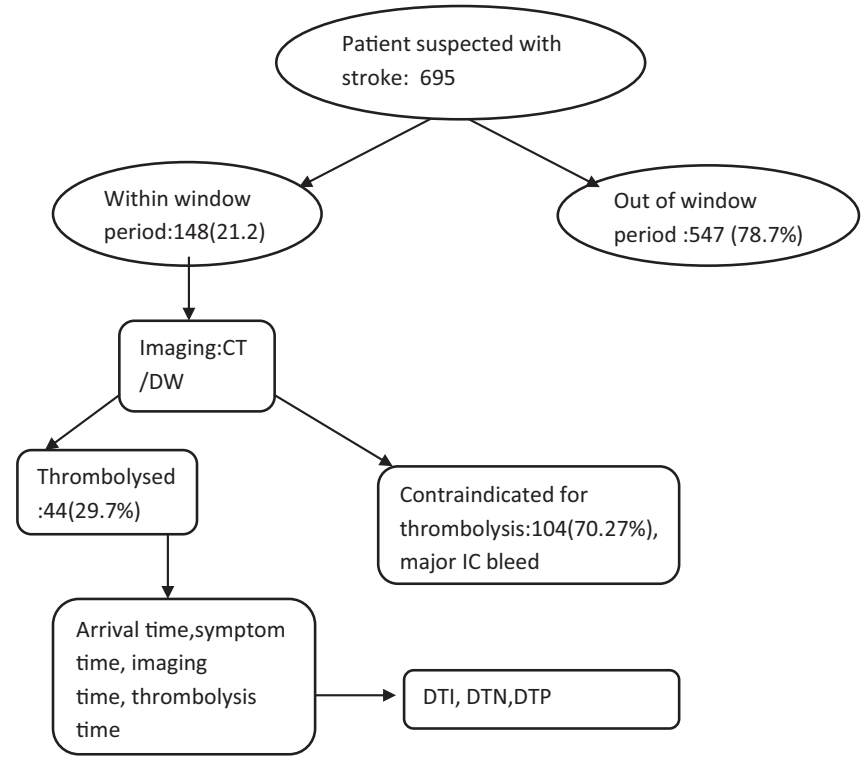

Figure I: Algorithm for patient presenting with stroke in emergency room (protocol used in the study)
The baseline characteristics of patient with acute ischemic stroke brought/admitted to ER, clinical features, arrival time (door time) to ER, severity of stroke, imaging time, radiological findings, contraindication for thrombolytic treatment, time of starting recombinant tissue plasminogen activator (rt-PA) and thereafter complications were recorded [Table 2].

The data abstracted were transferred to the SPSSTM 17.0 program (SPSS statistics is a software package used for statistical analysis. It is Statistical Package for Social Science and is produced by SPSS Inc.) of the computer for statistical analysis. The data were summarized as mean \pm standard deviation, median and percentages.

\section{Results}

Six hundred and ninety-five patients with symptoms of stroke were presented to our emergency department in the study period. Out of these five hundred and forty seven $(78.7 \%)$ were excluded as they had come out of window period that is, they had arrived $4.5 \mathrm{~h}$ after the onset of stroke symptoms. Thus, only 148 patients were included (21.2\%) [Figure 1]. Algorithm for patient presenting with stroke in ER (protocol used in the study).

Further after imaging of these one hundred and forty eight patients, one hundred four $(70.27 \%)$ were excluded. Sixty-two (59.6\%) had intra cerebral bleed, $1(0.9 \%)$ had hemoglobin $-3.1 \mathrm{~g}, 13(13 \%)$ of them had transient ischemic attack (neurological symptoms improved) and DWI images of these patient were normal. Relatives of $6(5.7 \%)$ patients refused to give consent for thrombolysis. 6 (5.7) patients were diagnosed to have metabolic de-rrangement (hypoglycemia, hyperglycemia, hyponatremia).

Three $(2.8 \%)$ patients had large middle cerebral artery infarct (MCA). Two (1.9\%) patients had acute ischemic attack secondary to mastoiditis. One $(0.9 \%)$ patients denied to undergo thrombolysis. Three $(2.8 \%)$ patients had recent stroke history. Other reasons for exclusion in our study were post-ictal status, financial problem, recent thrombolysis, recent surgery, and delay in contacting senior radiologist [Table 1].

Total $44(29.7 \%)$ patients with AIS were thrombolysed. Thirty-four $(79.5 \%)$ were male and nine $(20.45 \%)$ were female. Mean age of our patients was 57.32 years ranging from 24 to 79 . Co-morbid illness in the form of hypertension $6(13.6 \%)$, diabetes $2(4.5 \%)$, ischemic heart diseases (IHD) 2 (4.5\%), previous stroke CVA 
$3(6.5 \%),>1$ co-morbidity (HT/CVA/DM/IHD/ Hypothyriod) $14(31 \%)$, seizure $1(2 \%)$ and alcoholic liver diseases 1 (2\%) patients respectively. 1414 (31\%) patients had no co-morbidity [Table 2].

The mean blood pressure at admission was $140 / 90 \mathrm{mmHg}$. 11 (25\%) of them required labetalol before treatment. The baseline NIHSS score was 9.95 (2-21). The mean time for arrival of patients from onset of symptoms to hospital (STD) $1.23 \mathrm{~h}(15 \mathrm{~min}-3 \mathrm{~h})$. The mean door to neuro-physician time DTPt was $32 \mathrm{~min}$ (5 min-2.23 h). The mean DTIt, DTIt was 58 min ( 5 min $-4 \mathrm{~h}$ ). The mean DTNt $1.44 \mathrm{~h}$ (40 min-3.3 h) [Tables 3 and 4], [Figures 2-4].

\section{Discussion}

"Earlier the better" is stated in our guidelines. ${ }^{[8]}$ Analysis of our study clearly states that STDt, DTPt, DTIt, and DTNt time are significantly more. Thus, we had many hurdles in delivering thrombolysis therapy to these 44 patients. Only $7(15 \%)$ patients had DTNt $\leq 60 \mathrm{~min}$. Fonarrow et al. study showed only $26.6 \%$ of patients had DTNt of recommended period $<60 \mathrm{~min} \cdot{ }^{[3]}$

The problems/barriers in our study were categorized into three factors:

\section{Pre-hospital}

Mean symptom to door time was 83 min (median: 69). Lack of awareness of stroke symptoms in our community was main cause in delay.

Inaccessiblity to emergency medical services have contributed to delay. Poor recognition of stroke signs, especially in older patients caused delay in arrival time to hospital. ${ }^{[2]}$ Public and emergency medical services staff education play important role in shortening the pre hospital period. ${ }^{[4]}$

\section{Hospital factors}

In our study-door to physician, door to imaging and door to needle time were significantly ore compared to standard recommendations (AHA) [Table 3].

There was lack of handling stroke patients with high priority at each level ER, imaging unit, stroke unit. Thus lack of triaging stroke patient at all level of intervention was our weakest point. There was delay in informing reference to neuro physician in two of our patients. Education of emergency medical services of stroke symptoms will help to triage stroke patient. ${ }^{[5]}$ In one of the patients the on call doctor was very busy attending emergency calls so causing increase in DTPt time. The concept of having second on call doctor who takes care only of patients with acute stroke has being recommended by Kobayashi et al. ${ }^{[2]}$

Table I: Distribution of contraindications for thrombolytic therapy of patients

\begin{tabular}{lcc}
\hline Contraindications & $\boldsymbol{n}$ & $\mathbf{( \% )}$ \\
\hline Admission beyond window period & 547 & 78.7 \\
Intracerebral bleed (including SDH) & 62 & 41.8 \\
TIA & 13 & 9.45 \\
Relatives refusal & 6 & 5.7 \\
Metabolic encephalopathy & 6 & 5.7 \\
Recent CVA & 3 & 2.8 \\
Large MCA infarct & 3 & 2.8 \\
Drug over-dose & $\mathrm{I}$ & 0.9 \\
Epilepsy & $\mathrm{I}$ & 0.9 \\
Financial & 2 & 1.9 \\
Recently thrombolysed & $\mathrm{I}$ & 0.9 \\
Mastoiditis/meningitis & 2 & 1.9 \\
Recent surgery & $\mathrm{I}$ & 0.9 \\
Patient refusal & $\mathrm{I}$ & 0.9 \\
Demyelinating lesion & $\mathrm{I}$ & 0.9 \\
Low haemoglobin <3.I g & $\mathrm{I}$ & 0.9 \\
\hline CVA: Cerebro-vascular accident; TIA: Transient ischemic attack; SDH: Subdural
\end{tabular}

CVA: Cerebro-vascular accident; TIA: Transient ischemic attack; SDH: Subdural hematoma; MCA: Middle cerebral artery

Table 2: Baseline clinical characteristics of thrombolysed patients $(n=44)$

\begin{tabular}{|c|c|}
\hline Variables & $n(\%)$ \\
\hline Age (years), median (IQR) & $\begin{array}{c}\text { Male-55 (median: 56), } \\
\text { female-62.6 (median: 69) }\end{array}$ \\
\hline Male/female $(n)$ & $35 / 9$ \\
\hline No co-morbidity $n$ (\%) & $14(3 \mid)$ \\
\hline \multicolumn{2}{|l|}{ Co-morbidity } \\
\hline Hypertension $n(\%)$ & $7(15)$ \\
\hline Diabetes $n(\%)$ & $2(4.5)$ \\
\hline IHD (\%) & $2(4.5)$ \\
\hline Previous stroke $n(\%)$ & $3(6.8)$ \\
\hline More than one co-morbidity $n(\%)$ & $14(31)$ \\
\hline \multicolumn{2}{|l|}{ (HT/IHD/DM/CVA/hypothyroid) } \\
\hline Alcoholic liver disease & I (2) \\
\hline Seizure disorder & I (2) \\
\hline GCS score (mean) & 12.5 \\
\hline \multicolumn{2}{|l|}{ NIHSS, $n(\%)$} \\
\hline$\leq 5$ & II (25) \\
\hline $6-15$ & $26(59)$ \\
\hline $16-25$ & $7(15)$ \\
\hline $\begin{array}{l}\text { Stroke onset to arrival time (min) } \\
\text { (symptom to door time) mean (SD) }\end{array}$ & $83(47)$ \\
\hline Systolic blood pressure (mmHg), median (IQR) & $140(130-175)$ \\
\hline Diastolic blood pressure $(\mathrm{mmHg})$, median (IQR) & $90(80-110)$ \\
\hline Patients requiring labetelol $n(\%)$ & II (25) \\
\hline
\end{tabular}

Table 3: Our study DTPt, DTIt and DTNt compared with AHA guideline interval

\begin{tabular}{lcc}
\hline Time & $\begin{array}{c}\text { Recommended } \\
\text { intervals }\end{array}$ & Study time intervals \\
\cline { 3 - 3 } & $\mathrm{Imin})$ & Mean (median); SD min \\
\hline DTPt & 45 & $32($ I5); SD 34 \\
DTIt & $<60$ & $58(50) ;$ SD-50 \\
DTNt & $104(100) ;$ SD-4I
\end{tabular}

SD: Standard deviation; DTPt: Door to Physician time; DTIt: Door to imaging time; DTNt: Door to needle time; AHA: American Heart Association 


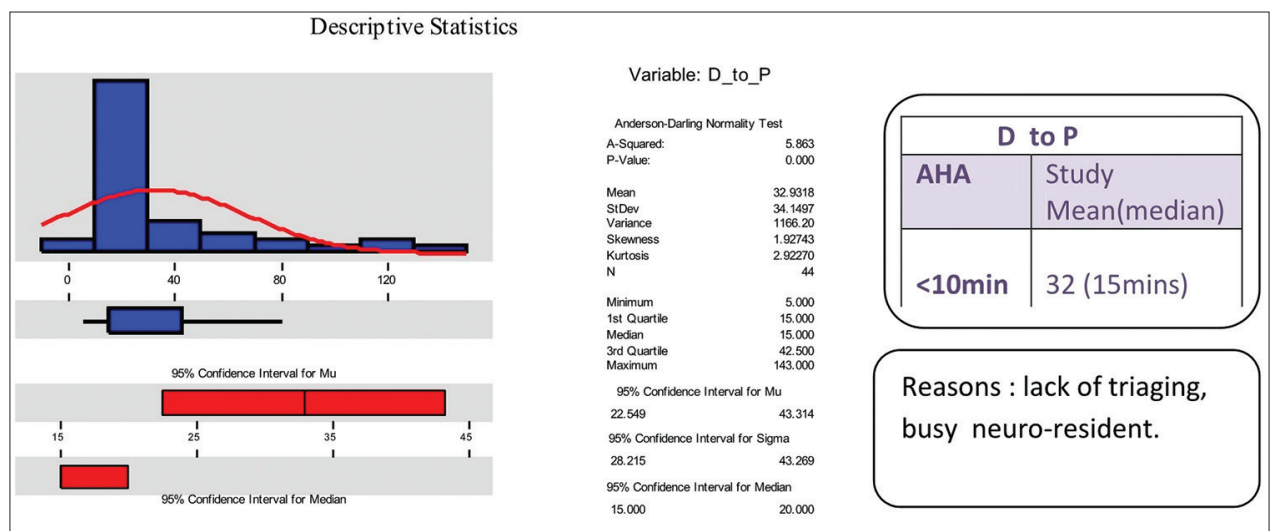

Figure 2: Door to physician time
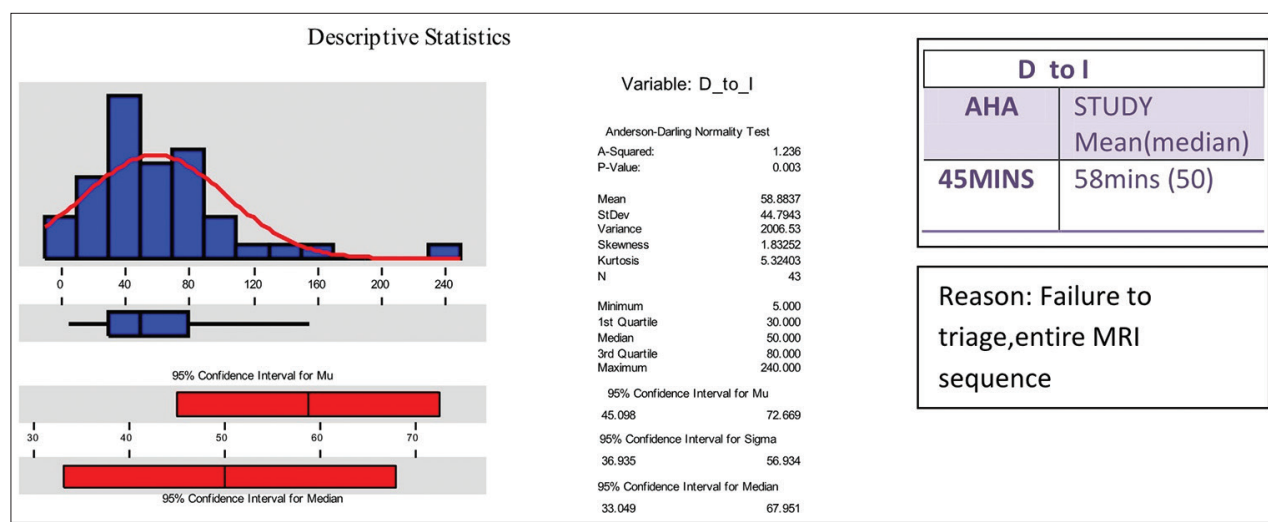

Reason: Failure to

triage,entire MRI

sequence

Figure 3: Door to imaging time (recommended standard time: $\leq 45 \mathrm{~min}$ )

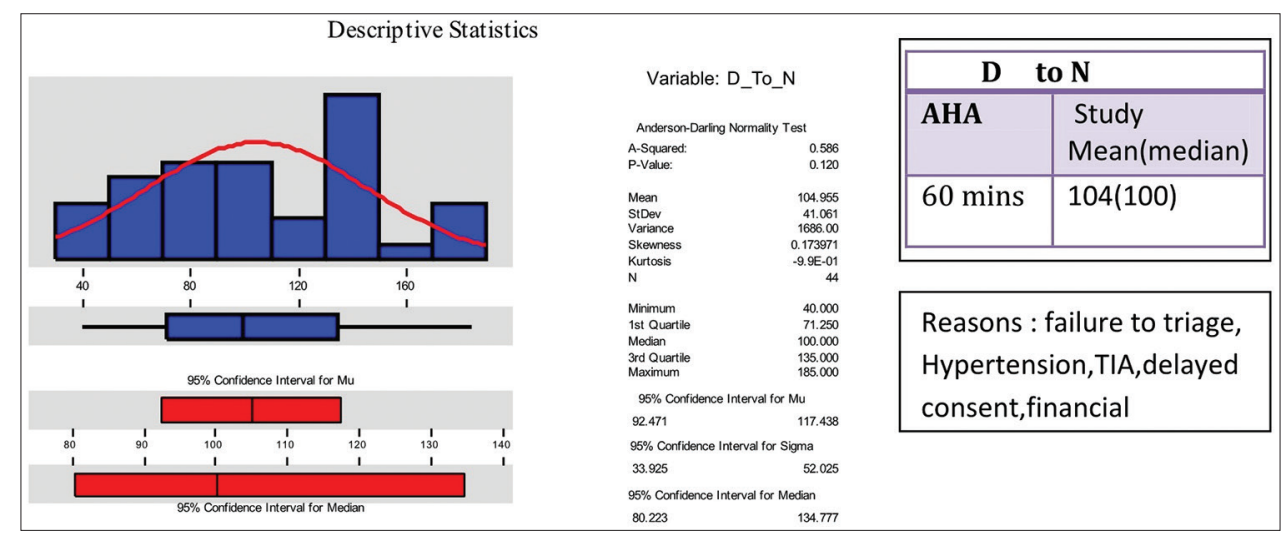

Figure 4: Door to needle time

\begin{tabular}{lc}
\hline Table 4: Number of patients thrombolysed per hour \\
\hline Time interval & Number of patients thrombolysed: $(\boldsymbol{n})$ \\
\hline$<60 \mathrm{~min}$ & 7 \\
$\mathrm{I}-2 \mathrm{~h}$ & 20 \\
$2-3 \mathrm{~h}$ & 16 \\
$3-4 \mathrm{~h}$ & $\mathrm{I}$ \\
\hline
\end{tabular}

Lack of triaging at radiology unit and performing entire sequences of magnetic resonance imaging (MRI) scan lead to increase in DTIt. Thus again prioritizing the stroke patient at radiology unit will help us to improvise ${ }^{[6]}$ Another important point is to perform only DWI image for deciding the thrombolysis therapy. ${ }^{[7]}$ DWI has high sensitivity (88-100\%) and specificity (95$100 \%$ ) for detecting infarcted regions, within minutes of onset of symptoms. ${ }^{[8]}$ Stephanie Paolini et al. study suggested " brain attack team MRI sequence" of $<10 \mathrm{~min}$ to confirm acute ischemia stroke and assess candidacy for IV-rtPA. ${ }^{[10]}$ Lack of triaging of bed for stroke patients resulted in increase in DTNt time. To prevent these delay we started thrombolysing AIS patient in ER. To prevent delay due to inavailability 
of drug, we have started keeping rtPA in our drug stock

\section{Patient factors}

Our DTNt was $104 \mathrm{~min}$ (door to needle time median - 100).

Patient with raised blood pressure requiring labetalol infusion caused delay. Relatives with geriatric patient (79 years) took longer time to give consent due to age of the patient and secondly due to financial burden. One of our patients had liver diseases so we had to wait for international normal ratio report for prothrombin time (international normalized ratio). One of our patients was in post-ictal state causing delay in thrombolysis. Recent guidelines removes this controversy if DWI images are done. In 2011 and 2012 transient ischemic attack patients were not thrombolysed, but in latter half of study DWI images helped us to prevent delay.

\section{Conclusions}

The barriers of thrombolysis in our study included:

- Lack of public awareness and Inaccessiblity to emergency medical services

- Lack of prioritizing triage system at ER, radiology unit and stroke unit

- Lack of a multi-disciplinary stroke care team..$^{[5,8]}$

A multi-disciplinary stroke care team consists of well-established emergency medical services, physicians, neurologist, nurses, radiology staff, neuro-radiologist, and pharmacist. Forming a one-call comprehensive "stroke code" will help in co-ordination at all level. Time to time audit of quality indicator of stroke code team may help to overcome the factors for delay in DTNt.

\section{References}

1 Tissue plasminogen activator for acute ischemic stroke. The national institute of Neurological Disorders and Stroke. rt-PA Stroke Study Group. N Eng J Med 1995;333:1581-7.

2 Adam Kobayashi, Marta S, Tomasz L,Anna C. Lack of experience of intravenous thrombolysis for acute ischemic stroke does not influence the proportion of patients treated. Emerg Med J 2007;24:96-99

3 Fonarow GC, Smith EE, Saver JL, Reeves MJ, Bhatt DL, Grau-Sepulveda MV, et al. Timeliness of tissue-type plasminogen activator therapy in acute ischemic stroke: Patient characteristics, hospital factors, and outcomes associated with door-to-needle times within 60 minutes: Circulation. 2011;123:750-8.

4 Edward CJ, Jeffrey LS, Harold PA, Jr, Askiel B, J.J. (Buddy) Connors et al; Guidelines for the Early Management of Patients With Acute Ischemic Stroke. A Guideline for healthcare professionals from the American Heart Association/American Stroke Association. Stroke 2013;44Nandigam K, Narayan SK, Elangovan S, Dutta TK, Sethuraman KR, Das AK. Feasibility of acute thrombolytic therapy for stroke. Neurol India 2003;51:470-3.

5 Sedat K, Emine D, Melek K, Abdullah SG, Said B. Limitations in Thrombolytic Therapy in acute Ischemic stroke: Pak J Med Sci 2012;284:586-590.

6 Rosalind F Yu, Ma. Cristina Z San Jose, Brenda MM, Micheal YO. Sources and reasons for delays in the care of acute stroke patients. Journal of the Neurological Sciences; Volume 199 2002: 49-54.

7 Nandigam K, Narayan S K, Elangovan S, Dutta T K, Sethuraman K R, Das A K .Feasibility of acute thrombolytic therapy for stroke. Neurol India 2003;51:470-3

8 Kavian G, Mohsen F, Ali P, Shirin G. Thrombolysis in Stroke Patients; Problems and Limitations. Iran J Med Sci 2010;35:145-8

9 Darshana A S, S Anand, Uday SL. Hyperacute stroke imaging: How much is enough? Indian J Radiology Imaging 2007;17:237-238.

10 Stephanie P, Joselyn B, Michael V, James W, Theodore F, Souvik $\mathrm{S}$ et al.Rapid Short MRI Sequence Useful in Eliminating Stroke Mimies Among Acute Stroke Patients Considered for Intravenous Thrombolysis.J Neurological Disorders 2013, 1:4

How to cite this article: Gurav SK, Zirpe KG, Wadia RS, Pathak MK, Deshmukh AM, Sonawane RV, et al. Problems and limitations in thrombolysis of acute stroke patients at a tertiary care center. Indian J Crit Care Med 2015;19:265-9.

Source of Support: Nil, Conflict of Interest: None declared. 